\title{
Confecção de Sistema Embarcado Dual-Banda Capaz de se Comportar como um Nó de uma Rede de Sensores sem fio
}

Title: Production of dual-band embedded system capable of behaving as a node in a wireless sensor network

\author{
Michelle Cavalcanti Maia \\ Escola Politécnica de Pernambuco \\ Universidade de Pernambuco \\ 50.720-001 - Recife, Brasil \\ me@michellemaia.com
}

\author{
Sérgio Campello Oliveira \\ Escola Politécnica de Pernambuco \\ Universidade de Pernambuco \\ 50.720-001 - Recife, Brasil \\ scampello@ecomp.poli.br
}

\begin{abstract}
Resumo Recentemente, as redes de sensores sem fio (RSSFs) têm sido bastante estudadas. Elas utilizam a retransmissão de pacotes para economizar a energia dos nós e garantir a entrega de pacotes ao destino. As RSSFs podem ser uma das poucas soluções tecnicamente viáveis em locais sem infraestrutura de telecomunicações disponível. Contudo, em ambientes urbanos, as RSSFs podem ser uma alternativa para evitar a dependência das redes de celular para o envio de informações. Este artigo descreve um sistema embarcado, com microcontrolador ARM Cortex-M0+, que incorpora dois módulos transceptores $R F$, baseados nas bandas ISM de $433 \mathrm{MHz}$ e de 2,4 $\mathrm{GHz}$. O dispositivo foi idealizado para se comportar como um nó de uma rede de sensores sem fio. Ele realiza a comunicação com os outros nós por meio dessas tecnologias sem fio. Para decidir a tecnologia a ser utilizada ao transmitir pacotes de dados, foi testado um algoritmo adaptado que atua fortemente no descarte de retransmissões desnecessárias. Dependendo das situações do ambiente, do sistema e da rede, a tecnologia sem fio a ser utilizada é escolhida e as informações são retransmitidas entre os nós sempre com dois objetivos: garantir a entrega dos pacotes e minimizar o consumo de energia.
\end{abstract}

Palavras-Chave: Sistema embarcado, Redes de sensores sem fio, Dual-banda ISM

\begin{abstract}
Recently, wireless sensor networks (WSNs) have been widely studied. They use the packets retransmission in order to save energy of the nodes and guarantee the packets delivery to the destination. WSNs can be one of the few technically viable solutions in locations without available telecommunications infrastructure. However, in urban environments, WSNs can be an alternative to avoid dependence on cellular networks for information sending. This article describes an embedded system with an ARM Cortex-MO+ based microcontroller, which incorporates two RF transceiver modules, based on the $433 \mathrm{MHz}$ and $2.4 \mathrm{GHz} I S M$ bands. The device was designed to behave as a node in a wireless sensor network. It communicates with the other nodes through these wireless technologies. In deciding the technology to be used to transmit data packets, an adapted algorithm was tested that operates strongly in the disposal of unnecessary retransmissions. Depending on the environmental, system and network conditions, the wireless technology to be used is selected and the information is retransmitted between the nodes always with two goals: to ensure the delivery of packages and to minimize power consumption.
\end{abstract}

Keywords: Embedded system, Wireless sensor networks, ISM dual-band 


\section{Introdução}

Uma rede de sensores sem fio é formada por um conjunto de nós capazes de transmitir informações através de enlaces de comunicação sem fio. Ela utiliza a retransmissão de pacotes para economizar a energia dos nós e garantir a entrega de pacotes ao destino. Uma das áreas de aplicação de RSSFs ocorre, em ocasião imperativa ou temporária, quando as instalações de redes de comunicação fixas são danificadas ou estão indisponíveis. Então, a RSSF é necessária por não depender de qualquer instalação de rede fixa e por sua característica de rápida autoorganização [1].

Uma possível aplicação para as redes de sensores sem fio é em serviços de venda com pontos eletrônicos que possuem diversos pontos de venda próximos e que podem funcionar como repetidores. Assim, suas atividades ficariam independentes ou menos dependentes das redes de celulares. Essas empresas que dependem da rede de celular para realizar suas atividades comerciais poderiam utilizar as RSSFs para reduzir custos operacionais, economizar energia da bateria dos equipamentos transmissores e ter flexibilidade na transmissão para garantir a entrega de pacotes de informações com o menor custo possível e menor atraso possível na entrega.

A solução de implementar uma RSSF poderia ser aplicada, por exemplo, no sistema de compartilhamento de bicicletas públicas que foram implementados nas grandes capitais brasileiras. Cada estação de bicicleta seria um nó que se comunicaria com as outras estações para levar a informação desejada até uma central. Adicionalmente ainda seria possível a instalação de circuitos eletrônicos de baixa potência nas bicicletas para utilizálas como retransmissores de dados e de suas próprias posições.

Diversas RSSFs foram implementadas utilizando apenas uma banda de comunicação. A banda de frequência ISM (industrial, scientific and medical) de $2,4 \mathrm{GHz}$ possui grande perda de percurso por metro, maior consumo e baixo poder de penetração em materiais de construção e quando chove, porém possui alta taxa de dados e utiliza antenas menores. A banda de frequência ISM de 433 $\mathrm{MHz}$ tem maior sensibilidade de recepção, menor perda de percurso e menor consumo. Porém é caracterizada por dispor de baixa taxa de dados, baixa qualidade de hardware disponível e as antenas tendem a ser maiores [2].

Para usufruir das qualidades e contornar as limitações, algumas aplicações já utilizam mais de uma banda de comunicação RF no sistema embarcado. $\mathrm{O}$ artigo de $\mathrm{A}$. Kim et al. [3] propõe a implementação de uma RSSF híbrida que utiliza tecnologia de comunicação sem fio baseada nas bandas de 2,4 GHz e $400 \mathrm{MHz}$. O trabalho investiga as diferenças de comunicação entre duas bandas no ambiente de um prédio. Então, foi indicado a utilização de nós com sensores portáteis, alimentados por bateria e com baixa taxa de dados, empregando a topologia estrela para se comunicar com o nó coordenador através da banda de $400 \mathrm{MHz}$. A topologia mesh é utilizada para a comunicação com uma taxa de dados veloz entre os nós coordenadores através da banda de 2,4 GHz.

O objetivo deste trabalho é propor um sistema embarcado capaz de se comportar como um nó de uma RSSF baseada em duas tecnologias que utilizam diferentes bandas RF ISM. A energia determina a longevidade e influencia as decisões práticas de uma rede de sensores, portanto, os nós da rede devem ter baixo consumo de energia. A transmissão de informação em nós sensores gasta mais energia que a implementação de cálculos pelo microcontrolador [4]. Então, na tentativa de redução do consumo de energia, primeiro o nó envia os dados através do módulo RF de $433 \mathrm{MHz}$, que possui menor alcance mas gasta menos energia. Caso o nó não receba confirmação que a mensagem foi enviada, o módulo RF de 2,4 $\mathrm{GHz}$ é utilizado para retransmitir os dados. Para assegurar a entrega dos pacotes à estação base e reduzir retransmissões desnecessárias também foi adaptado e testado um protocolo de estratégia de coordenaçãocomunicação unificada [5].

As próximas seções deste artigo estão organizadas da seguinte forma: a Seção 2 contém o projeto de hardware do sistema embarcado. A Seção 3 exibe o processo de desenvolvimento de firmware com as ferramentas utilizadas. A Seção 4 apresenta a implementação do protocolo de comunicação e os testes realizados. Por fim, a Seção 5 apresenta a conclusão do artigo e discute trabalhos futuros.

\section{Projeto de hardware}

Os projetos de hardware e firmware foram inspirados na plataforma de desenvolvimento Freedom, da Freescale [6]. Essa plataforma preenche os requisitos de utilizar microcontroladores com arquitetura ARM e de disponibilizar exemplos de projetos de hardware. Ainda possui suporte completo open source do desenvolvimento do firmware com edição e compilação do projeto, gravação da memória flash do microcontrolador e depuração do código.

As placas da plataforma Freedom utilizam os microcontroladores da família Kinetis [7], que possuem arquitetura de 32 bits com núcleo ARM (Cortex-M0+, CortexM4 e Cortex-M7) e alta performance para baixo consumo. Cada placa possui o sistema OpenSDA [8], programador e depurador serial, que faz uma ponte de comuni- 
cação entre um dispositivo USB host e o microcontrolador alvo. O projeto de hardware foi desenvolvido na ferramenta CAD Altium Designer [9].

\subsection{Visão geral}

Os principais elementos do sistema embarcado são: bloco de alimentação, bloco do microcontrolador, os blocos dos transceptores RF e o cartão de memória micro SD. O sistema está representado em diagramas de blocos com suas interconexões na Figura 1.

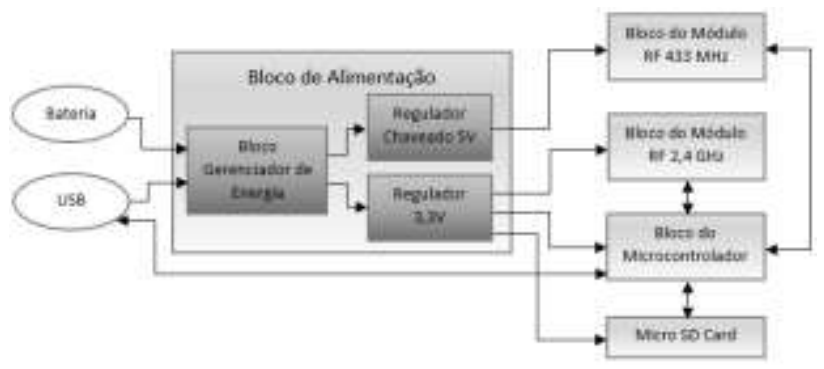

Figura 1: Diagrama de blocos do sistema.

\subsection{Bloco de alimentação}

O principal dispositivo do bloco gerenciador de energia é o CI BQ24075RGTT [10], que é um carregador de bateria Li-Ion de apenas 1 célula e gerenciador do caminho de energia do sistema. O esquemático com o CI é mostrado na Figura 2. O recurso de gerenciamento do caminho dinâmico de energia alimenta o sistema enquanto simultaneamente e independentemente carrega a bateria. Esse recurso reduz o número de ciclos de carga e descarga da bateria, permitindo o término adequado do carregamento da bateria.

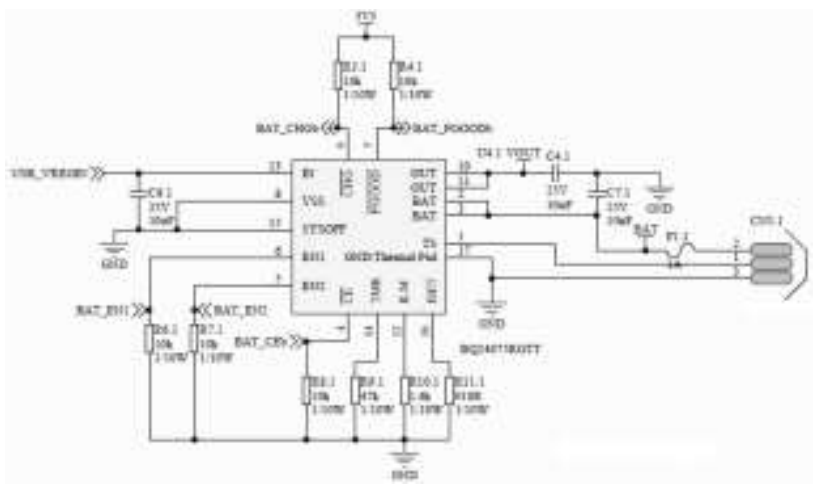

Figura 2: Circuito para carregar bateria Li-Ion e fornecer energia ao sistema com o CI BQ24075RGTT.

A tensão de entrada de $5 \mathrm{~V}$ do componente, USB VREGIN, é fornecida pela interface USB. Quando conectado à interface USB, a tensão de saída do componente, VOUT, é 4,4 V. Quando o componente está desconectado VOUT assume o valor da tensão da bateria. VOUT é a tensão de entrada do regulador linear de 3,3 V e do regulador chaveado de $5 \mathrm{~V}$.
O componente permite que a carga seja alimentada assim que ocorre uma conexão com uma fonte externa, mesmo com a bateria totalmente descarregada. A bateria também complementa o fornecimento de corrente quando a fonte externa não pode proporcionar as correntes de pico do sistema.

O dispositivo é importante porque respeita o complexo procedimento de carregar uma bateria Li-Ion. O processo ocorre em três fases: fase de pré-carga, fase de carga rápida com corrente constante e a fase de estabilização com tensão constante. A Figura 3 mostra o ciclo de carregamento da bateria completo. Na fase de pré-carga, a bateria é carregada com o valor da corrente igual a $10 \%$ do valor da corrente de carga rápida. Uma vez que a tensão da bateria atinge $3 \mathrm{~V}$, a bateria será carregada com a corrente constante de carga rápida. Quando a tensão da bateria atinge o valor final de $4,2 \mathrm{~V}$, ela é carregada com essa tensão final até atingir a carga completa.

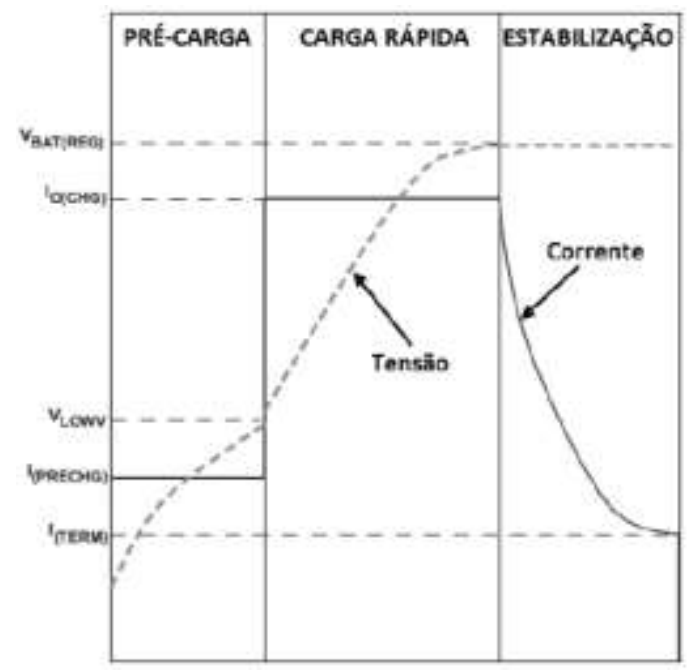

Figura 3: Ciclo de carregamento para bateria Li-Ion de 3,7 V gerenciado pelo CI BQ24075RGTT.

Existe também um controle interno do CI que monitora a temperatura através da conexão com um termistor NTC. Se a temperatura estiver fora da faixa monitorada entre $0^{\circ} \mathrm{C}$ e $50^{\circ} \mathrm{C}$, o carregamento da bateria é suspenso. $\mathrm{O}$ limite do tempo de carregamento da bateria e o valor da corrente de carga rápida são programáveis usando resistores externos. O microcontrolador determina o limite de corrente de entrada através de uma conexão com o CI carregador de bateria, utilizando 2 pinos digitais.

Para alimentar o microcontrolador [11], o módulo RF de 2,4 GHz [12], o cartão micro SD [13] e o LED RGB [14], foi utilizado o CI SPX3819M5-L-3-3/TR [15], regulador linear de 3,3 V com capacidade de $500 \mathrm{~mA}$. Para alimentar o bloco do módulo RF de $433 \mathrm{MHz}$ [16], foi utilizado o CI NCP1400ASN50T1G [17], regulador chaveado tipo Boost para garantir o nível de tensão de $5 \mathrm{~V}$ e 
corrente máxima de $100 \mathrm{~mA}$, mesmo quando o sistema está alimentado apenas pela bateria Li-Ion de 3,7 V.

\subsection{Bloco do microcontrolador}

As características do microcontrolador MKL25Z128VLK4 relevantes para o projeto são apresentadas na Tabela 1 .

Além do microcontrolador, os circuitos do bloco são: interface de gravação SWD, circuito de reset e circuito oscilador com cristal de $8 \mathrm{MHz}$. A frequência máxima de $48 \mathrm{MHz}$ é utilizada com o auxílio do PLL do microcontrolador.

\begin{tabular}{|c|c|}
\hline Característica & Descrição \\
\hline $\begin{array}{l}\text { Núcleo do } \\
\text { Processador }\end{array}$ & ARM Cortex-M0+ \\
\hline $\begin{array}{l}\text { Tamanho do } \\
\text { Núcelo }\end{array}$ & 32-bit \\
\hline $\begin{array}{l}\text { Frequência } \\
\text { máxima }\end{array}$ & $48 \mathrm{MHz}$ \\
\hline Conectividade & $\begin{array}{l}\text { I2C, LIN, SPI, UART/USART, } \\
\text { USB OTG }\end{array}$ \\
\hline Periféricos & $\begin{array}{l}\text { Brown-out Detect/Reset, DMA, } \\
\text { LVD, POR, PWM, WDT, RTC }\end{array}$ \\
\hline Número de $\mathrm{E} / \mathrm{S}$ & 66 \\
\hline $\begin{array}{l}\text { Memória de } \\
\text { programa }\end{array}$ & $128 \mathrm{~KB}(128 \mathrm{~K} \times 8) \quad$ FLASH \\
\hline Tamanho da RAM & $16 \mathrm{~K} \times 8$ \\
\hline $\begin{array}{l}\text { Tensão de } \\
\text { alimentação }\end{array}$ & $1,71 \mathrm{~V} \sim 3,6 \mathrm{~V}$ \\
\hline $\begin{array}{l}\text { Conversores de } \\
\text { dados }\end{array}$ & $\mathrm{A} / \mathrm{D} 14 \mathrm{x} 16 \mathrm{~b}, \mathrm{D} / \mathrm{A} 1 \mathrm{x} 12 \mathrm{~b}$ \\
\hline
\end{tabular}

Tabela 1: Características do microcontrolador MKL25Z128VLK4.

\subsection{Bloco dos módulos RF}

Ambos os módulos foram selecionados por respeitarem os critérios de baixo custo (menos de $\mathrm{R} \$ 10,00$ cada módulo RF, excluindo o valor e o processo de soldagem da antena), disponibilidade de compra no mercado brasileiro e vasto material didático disponível on-line. Os módulos RF são encaixados em conectores da placa de circuito impresso do sistema embarcado projetado.

O bloco RF de $433 \mathrm{MHz}$ é dividido em 2 partes: os módulos transmissor (com tamanho de $30 \mathrm{~mm} \times 12 \mathrm{~mm}$ ) e receptor (com tamanho de $20 \mathrm{~mm} \times 20 \mathrm{~mm}$ ), mostrados na Figura 4. Devido ao menor custo, antenas helicoidal de um quarto de onda são utilizadas em ambos os módulos. As características dos módulos são apresentadas na Tabela 2.
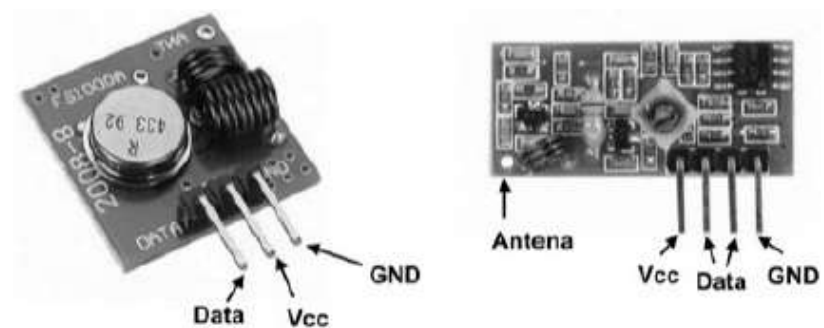

Figura 4: Módulos receptor e transmissor $433 \mathrm{MHz}$.

Foram utilizados resistores como divisor de tensão para fazer o casamento de sinais entre o receptor, modelo MX-FS-03V alimentado com $5 \mathrm{~V}$, e o microcontrolador, alimentado com 3,3 V. O transmissor, modelo MX-FS$03 \mathrm{~V}$, é conectado diretamente ao microcontrolador porque é compatível com 3,3 V. O microcontrolador recebe e envia informações através de 2 pinos GPIO, um como entrada e outro como saída.

Os módulos do bloco RF de $433 \mathrm{MHz}$ não possuem modo sleep. O microcontrolador deve monitorar continuamente o pino de conexão com o módulo receptor para saber se recebeu uma mensagem. Para diminuir o consumo de corrente, é recomendado ligar o módulo transmissor apenas na hora de transmitir informação.

\begin{tabular}{l|l}
\hline \multicolumn{1}{c|}{ Característica } & \multicolumn{1}{c}{ Descrição } \\
\hline Banda ISM de frequência & $433 \mathrm{MHz}$ \\
\hline Modulação & ASK \\
\hline Taxa de transferência máxima & $5 \mathrm{kbit} / \mathrm{s}$ \\
\hline Alcance máximo & $200 \mathrm{~m}$ \\
\hline Corrente módulo receptor & $4 \mathrm{~mA}$ \\
\hline Sensibilidade de recepção & $-105 \mathrm{~dB}$ \\
\hline Potência módulo transmissor & $10 \mathrm{~mW}$ \\
\hline Tensão de alimentação & $3,5 \mathrm{~V} \sim 12 \mathrm{~V}$ \\
\hline
\end{tabular}

Tabela 2: Características dos módulos RF de $433 \mathrm{MHz}$.

O bloco RF 2,4 GHz é formado por uma placa (com tamanho de $40 \mathrm{~mm} \times 15 \mathrm{~mm}$ ) com o transceptor nRF24L01+, da empresa Nordic Semiconductor, e antena externa LNA com ganho de $2 \mathrm{dBi}$. As características do módulo são apresentadas na Tabela 3.

O módulo RF 2,4 GHz possui modo sleep, importante para diminuir o consumo de corrente do sistema. Para receber mensagens é preciso está no modo de recepção para que o CI nRF24L01+ monitore o canal RF. O CI possui um pino de interrupção que informa ao microcontrolador quando uma mensagem foi recebida (característica útil quando é preciso colocar o microcontrolador em modo de economia de energia). Para transmitir mensagens é necessário está no modo transmissão. O microcontrolador envia comandos para selecionar o modo desejado 
e apenas é possível assumir um modo por vez. A comunicação entre o módulo RF $2,4 \mathrm{GHz}$ e o microcontrolador é realizada através de uma interface SPI.

\begin{tabular}{l|l}
\hline \multicolumn{1}{c|}{ Característica } & \multicolumn{1}{c}{ Descrição } \\
\hline Banda ISM de frequência & $2,4 \mathrm{GHz}$ \\
\hline Modulação & $\mathrm{GFSK}$ \\
\hline Taxa de transferência máxima & $2 \mathrm{Mbit} / \mathrm{s}$ \\
\hline Alcance máximo & $1 \mathrm{~km}$ \\
\hline Corrente modo transmissão & $145 \mathrm{~mA}$ \\
\hline Corrente modo recepção & $45 \mathrm{~mA}$ \\
\hline Corrente modo sleep & $4,3 \mathrm{uA}$ \\
\hline Sensibilidade de recepção & $-104 \mathrm{dBm}$ \\
\hline Tensão de alimentação & $1,9 \mathrm{~V} \sim 3,6 \mathrm{~V}$ \\
\hline
\end{tabular}

Tabela 3: Características do módulo RF de 2,4 GHz.

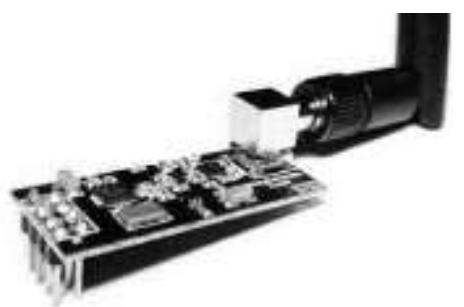

Figura 5: Módulo nRF24L01 transceptor 2,4 GHz.

O CI nRF24L01+ possibilita a utilização da tecnologia ShockBurst [12] com acelerador de protocolo por hardware, que habilita montagem automática de pacote, confirmação de envio e retransmissões automáticas de mensagens. A tecnologia permite a implementação de sistemas de baixo consumo e de alta performance com microcontroladores de baixo custo [4].

\subsection{Periféricos}

Todas as mensagens recebidas e enviadas pelo sistema são gravadas em um arquivo de registro txt, armazenado em um cartão de memória micro SD. Detalhes como horário do evento e qual módulo RF foi utilizado também são gravados. O sistema deixa registrado quando ocorreu falha ao enviar uma mensagem. A comunicação entre o cartão e o microcontrolador é realizada através de uma interface SPI.

As informações armazenadas no cartão de memória podem ser utilizadas para checar a eficiência do protocolo de comunicação escolhido e proporcionar melhorias futuras. Para uma implementação comercial do sistema, essa funcionalidade pode ser desligada para economizar energia e reduzir os custos de fabricação.

A interface USB é a fonte principal de alimentação do sistema. Além disso, há a possibilidade de conectar o sistema a uma porta USB do computador para visualizar as mensagens enviadas e recebidas pelo nó conectado em tempo real. É possível ajustar o horário do nó conectado e enviar essa informação para sincronizar o periférico RTC do microcontrolador dos outros nós (através dos módulos de RF).

Há também um LED RGB indicador de estado e um conector que possibilita acesso externo a uma interface SPI, duas interfaces UART, uma interface I2C e um par diferencial do módulo ADC. Dessa forma, o módulo pode ser conectado a vários sensores com interfaces de comunicação diferentes.

\subsection{Placa de circuito impresso}

Três placas de circuito impresso com tamanho de $50 \mathrm{~mm} \times 50 \mathrm{~mm}$ foram produzidas. A face inferior de uma placa é mostrada na Figura 6 e a face superior na Figura 7.

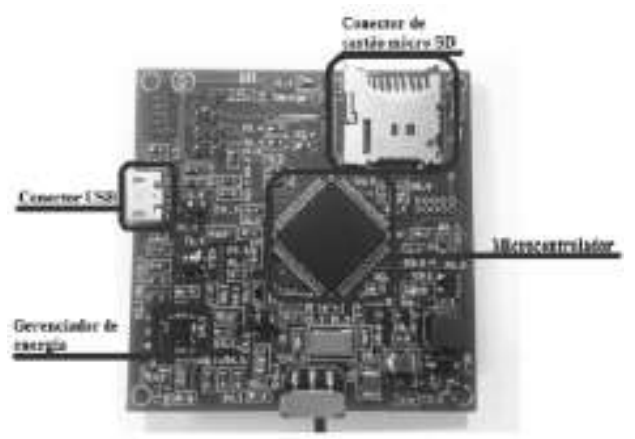

Figura 6: Face inferior da placa.

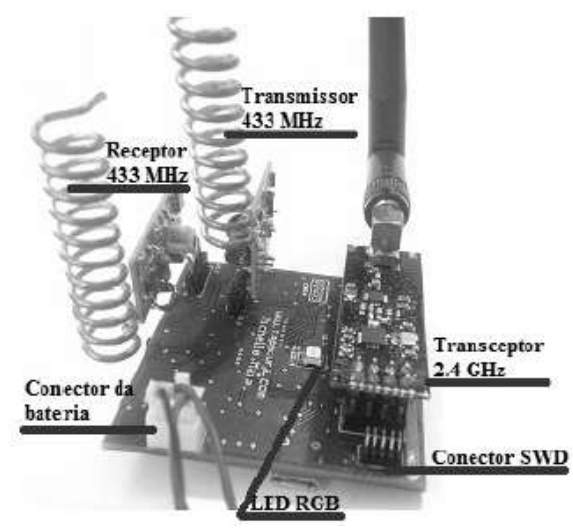

Figura 7: Face superior da placa.

\section{Processo de desenvolvimento de Firmware}

A Freescale oferece suporte de firmware com a ferramenta de desenvolvimento Kinetis Design Studio IDE [18]. A IDE open source (baseada na estrutura do Eclip- 
se), permite edição robusta (sem limitação de código), inclui compilador GCC e depurador GDB. Ainda possui o plug-in Processor Expert [19], capaz de criar os drivers dos periféricos do microcontrolador com alguns cliques do mouse. O código do projeto foi desenvolvido na plataforma Kinetis, com a linguagem C (por ser uma linguagem estruturada, portável e gerar códigos compactos).

O código do firmware foi implementado com auxílio do sistema operacional em tempo real FreeRTOS [20], para aumentar o controle sobre as tarefas realizadas pelo microcontrolador. O sistema embarcado executa funções em tempo real e deve concluir dentro de um determinado limite de tempo crítico. Um erro pode resultar na falha absoluta do sistema [21]. O FreeRTOS é projetado para ser pequeno e simples (entre $4 \mathrm{~K}$ e $9 \mathrm{~K}$ bytes). O núcleo do kernel consiste em três arquivos $\mathrm{C}$. O sistema operacional fornece métodos para múltiplas tarefas (com diferentes prioridades), semáforos e temporizadores em software. Há 4 opções de alocação de memória. Além de tudo, a plataforma é open source, é portável para vários sistemas embarcados e possui rica documentação.

\section{Implementação do protocolo de co- municação e testes}

Para teste de comunicação inicial, uma rede de sensores foi montada utilizando três módulos. O protocolo de comunicação para Veículos Aéreos Não Tripulados (VANTs) [5] foi adotado com algumas modificações.

Nesse experimento foram reduzidos alguns bits de informação dos elementos dos pacotes pois o tamanho máximo do pacote enviado pelo módulo RF $2,4 \mathrm{GHz}$ é 32 bytes. Caso se desejasse manter o tamanho dos elementos, a mensagem poderia ser enviada em 2 pacotes.

Os pacotes enviados contêm coordenadas GPS e o sistema desenvolvido não possui módulo GPS. A solução para testar o protocolo foi utilizar coordenadas simuladas.

Cada nó foi modelado com uma característica: um nó transmissor, um nó repetidor e um nó base. $\mathrm{O}$ protocolo de comunicação conta com 4 tipos de pacotes. O nó transmissor foi modelado, enviando pacotes de informação nos formatos TIPO I e TIPO II, mostrados nas Tabela 4 e Tabela 5 respectivamente.

O pacote TIPO I é enviado para questionar o status de conexão com a base. Os campos ID, Tipo e $\mathrm{N}^{\circ}$ do VANT de origem identificam unicamente o pacote. $O$ pacote contém também as coordenadas GPS simuladas do nó.

\begin{tabular}{c|c|c|c|c|c|c|c}
\hline ID & Tipo & $\begin{array}{c}\mathbf{N}^{\circ} \\
\text { VANT } \\
\text { Origem }\end{array}$ & $\begin{array}{c}\mathbf{N}^{\circ} \\
\text { VANT } \\
\text { Retrans }\end{array}$ & $\begin{array}{c}\text { Lat } \\
\text { VANT } \\
\text { Origem }\end{array}$ & $\begin{array}{c}\text { Long } \\
\text { VANT } \\
\text { Origem }\end{array}$ & $\begin{array}{c}\text { Lat } \\
\text { VANT } \\
\text { Retrans }\end{array}$ & $\begin{array}{c}\text { Long } \\
\text { VANT } \\
\text { Retrans }\end{array}$ \\
\hline 6 & 2 & 8 bits & 8 bits & $\begin{array}{c}32 \\
\text { bits }\end{array}$ & $\begin{array}{c}32 \\
\text { bits }\end{array}$ & 32 bits & 32 bits \\
\hline bits & bits & bits &
\end{tabular}

Tabela 4: Formato do pacote TIPO I, questiona o status com a base.
O pacote TIPO II é enviado para informar à base a detecção de um alvo. Os campos com as coordenadas GPS simuladas de um alvo são adicionadas ao pacote.

\begin{tabular}{c|c|c|c|c|c|c|c|c|c}
\hline ID & $\begin{array}{c}\text { Ti- } \\
\text { po }\end{array}$ & $\begin{array}{c}\mathbf{N}^{\circ} \\
\text { VANT } \\
\text { Ori- } \\
\text { gem }\end{array}$ & $\begin{array}{c}\mathbf{N}^{\mathbf{0}} \\
\text { VANT } \\
\text { Re- } \\
\text { trans }\end{array}$ & $\begin{array}{c}\text { Lat } \\
\text { VANT } \\
\text { Ori- } \\
\text { gem }\end{array}$ & $\begin{array}{c}\text { Long } \\
\text { VANT } \\
\text { Ori- } \\
\text { gem }\end{array}$ & $\begin{array}{c}\text { Lat } \\
\text { VANT } \\
\text { Re- } \\
\text { trans }\end{array}$ & $\begin{array}{c}\text { Long } \\
\text { VANT } \\
\text { Re- } \\
\text { trans }\end{array}$ & $\begin{array}{c}\text { Lat } \\
\text { Al- } \\
\text { vo }\end{array}$ & $\begin{array}{c}\text { Lon } \\
\mathbf{g} \\
\text { Al- } \\
\text { vo }\end{array}$ \\
\hline $\begin{array}{c}6 \\
\text { bit } \\
\text { s }\end{array}$ & $\begin{array}{c}2 \\
\text { bit }\end{array}$ & $\begin{array}{c}8 \\
\text { sits }\end{array}$ & $\begin{array}{c}8 \\
\text { bits }\end{array}$ & $\begin{array}{c}32 \\
\text { bits }\end{array}$ & $\begin{array}{c}32 \\
\text { bits }\end{array}$ & $\begin{array}{c}32 \\
\text { bits }\end{array}$ & $\begin{array}{c}32 \\
\text { bits }\end{array}$ & $\begin{array}{c}32 \\
\text { bit } \\
\text { sit }\end{array}$ & $\begin{array}{c}32 \\
\text { bit } \\
\text { sit }\end{array}$ \\
\hline
\end{tabular}

Tabela 5: Formato do pacote TIPO II, informa detecção de alvo.

O nó transmissor envia pacotes TIPO I ou TIPO II no intervalo de tempo de $5 \mathrm{~s}$, como mostra a Figura 8. O ciclo do teste é iniciado utilizando o módulo de 433 MHz. São enviadas várias mensagens alterando a localização GPS simulada do nó transmissor. Se no intervalo definido o nó transmissor não recebe a resposta do nó base, ele é considerado desconectado da rede e adota o módulo de 2,4 GHz para enviar nova mensagem.

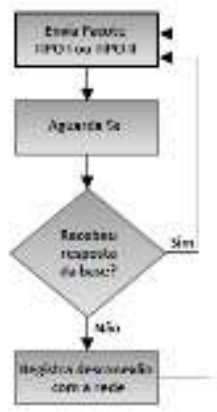

Figura 8: Fluxograma do comportamento do nó transmissor.

O nó repetidor foi modelado apenas para retransmitir pacotes utilizando os critérios do fluxograma mostrados na Figura 9 para decidir se descarta ou repassa o pacote com a informação. Como as coordenadas GPS são apenas simuladas, o nó repetidor e o nó base recebem todas as mensagens enviadas pelo nó transmissor. Então, as mensagens que estariam fora do raio de comunicação são ignoradas via software. O raio de comunicação (Rcom) de $1 \mathrm{~km}$ é considerado para o módulo de $2,4 \mathrm{GHz}$ e Rcom igual a $200 \mathrm{~m}$ é considerado para o módulo de $400 \mathrm{MHz}$.

Na primeira vez que o pacote é recebido, o nó repetidor o empilha. Depois, o teste de distância é executado. Se a distância for maior que Rcom/2 (metade do raio de comunicação), o repetidor repassa o pacote imediatamente. Se a distância for menor que Rcom $/ 2$, o nó inicia um contador de $2 \mathrm{~s}$. Se uma cópia do pacote for recebida nesse intervalo, o pacote não é transmitido, mas mantido na pilha. Se não receber uma cópia, o pacote é repassado. Quando o pacote é passado adiante os campos de $\mathrm{N}^{\circ} \mathrm{e}$ das coordenadas GPS do VANT retransmissor são preenchidos com o número que identificam o nó repetidor e suas coordenadas GPS simuladas. A mensagem é enviada na mesma banda de frequência que o sinal foi recebido. 


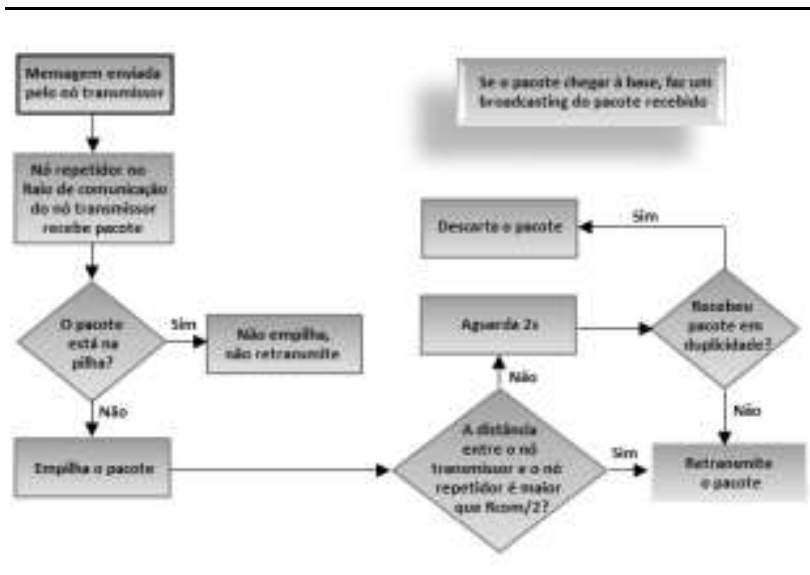

Figura 9: Fluxograma do comportamento do nó repetidor.

O nó base foi modelado enviando pacotes de respostas nos formatos TIPO III, que é a resposta ao pacote TIPO I, e TIPO IV, que é a resposta ao pacote TIPO II. Os formatos dos pacotes são mostrados nas Tabela $6 \mathrm{e}$ Tabela 7. A mensagem é sempre enviada pelo módulo de $2,4 \mathrm{GHz}$ e é considerado que a base tem alcance suficiente para atingir todos os outros nós.

\begin{tabular}{c|c|c|c|c}
\hline ID & Tipo & $\mathbf{N}^{\circ}$ VANT Origem & $\begin{array}{c}\text { Lat VANT } \\
\text { Origem }\end{array}$ & $\begin{array}{c}\text { Long VANT } \\
\text { Origem }\end{array}$ \\
\hline 6 bits & 2 bits & 8 bits & 32 bits & 32 bits \\
\hline
\end{tabular}

Tabela 6: Formato do pacote TIPO III, resposta da base ao pacote TIPO I.

\begin{tabular}{c|c|c|c|c|c|c}
\hline ID & Tipo & $\begin{array}{c}\mathbf{N}^{\circ} \text { VANT } \\
\text { Origem }\end{array}$ & $\begin{array}{c}\text { Lat VANT } \\
\text { Origem }\end{array}$ & $\begin{array}{c}\text { Long } \\
\text { VANT } \\
\text { Origem }\end{array}$ & Lat Alvo & Long Alvo \\
\hline 6 & $\begin{array}{c}2 \\
\text { bits }\end{array}$ & 8 bits & 32 bits & 32 bits & 32 bits & 32 bits \\
\hline
\end{tabular}

Tabela 7: Formato do pacote TIPO IV, resposta da base ao pacote TIPO II.

Como descrito anteriormente, todas as informações enviadas e recebidas pelo nó são gravadas no cartão de memória micro SD com o horário do evento e módulo de comunicação utilizado. Quando o nó é conectado ao computador, essas informações podem ser visualizadas em tempo real através da conexão USB CDC.

O LED RGB também foi utilizado para ter uma visualização do comportamento da rede em tempo real. Quando um nó envia informação pelo módulo de $433 \mathrm{MHz}$, o LED emite a cor amarela. Quando recebe informação pelo módulo de $433 \mathrm{MHz}$, o LED emite a cor verde. O LED emite cor roxa, quando o nó envia dados pelo módulo de 2,4 GHz. O LED emite a cor azul, quando o nó recebe dados pelo módulo de $2,4 \mathrm{GHz}$.

$\mathrm{O}$ experimento foi filmado e pode ser visualizado na internet [22].

\section{Conclusão e trabalhos futuros}

Este artigo propôs a confecção de um sistema embarcado com um bloco de alimentação robusto, microcontrolador, 2 transceptores RF nas bandas ISM de $433 \mathrm{MHz}$ e
2,4 GHz e o cartão de memória. O sistema foi utilizado para criar uma RSSF e testar o protocolo que assegura a entrega dos pacotes à estação base e reduz retransmissões desnecessárias.

Os testes de transmissão entre os nós da RSSF foram realizados com sucesso nas duas faixas de frequência utilizadas. O registro de envio e recepção dos pacotes funcionou e pode ser usado para análises estatísticas e melhorias futuras.

As próximas etapas do projeto são: implementar o protocolo de comunicação para VANTs completo para cada nó da rede; verificar o comportamento da rede com maior número de nós inseridos; fazer testes de distância, consumo e taxa de sucesso na entrega de pacotes; adicionar módulo GPS para obter informações reais de localização.

A última etapa é acrescentar um modem GSM, após estudos do aumento do consumo de energia da bateria ao acrescentar esse item ao projeto. $\mathrm{O}$ modem é para ser utilizado em caso extremo, quando um nó ficar incomunicável com a rede de sensores, os dados serem enviados pela rede de celular.

\section{Referências}

[1] W. Liu, H. Chen, M. Chen. A Survey of Wireless Sensor Networks. Proceedings of Conference on Dependable Computing, páginas 305307, Novembro 2010.

[2] Myk. Dormer. Choice of frequency band can realy make a difference. Electronics World, Vol. 114, edição 1866, páginas 16-18, Junho 2008.

[3] A. Kim, J. Han, T. Yu, D. S. Kim. Hybrid wireless sensor network for building energy management systems based on the $2.4 \mathrm{GHz}$ and 400 $\mathrm{MHz}$ bands. In Information Systems, Elsevier, páginas 320-326, Março 2014.

[4] S. S. Sonavane, V. Kumar, B. P. Patil. Designing wireless sensor network with low cost and low power. In 16th IEEE International Conference on Networks (ICON 2008), Dezembro 2008.

[5] S. C. Oliveira, G. A. Duarte, H. B. da Cunha Júnior. Unified Coordination-Communication Strategy to Swarm Controlled Mobile Wireless Sensor Network. In IEEE Latim America Transactions, VOL. 12, NO. 5, páginas 951-956,

[6] Freescale Semiconductor, Inc. Freescale Freedom Development Boards. http://www.freescale.com/webapp/sps/site/overv iew.jsp?code=FREDEVPLA , Julho 2015. 
[7] Freescale Semiconductor, Inc. Kinetis Low Power 32-bit Microcontroller (MCUs) based on ARM Cortex-M Cores. http://www.freescale.com/products/armprocessors/kinetis-cortex-m:KINETIS ，Julho 2015.

[8] Freescale Semiconductor, Inc. OpenSDA Serial and Debug Adapter.

http://www.freescale.com/tools/embeddedsoftware-and-tools/run-time-software/kinetissoftware-and-tools/ides-for-kinetismcus/opensda-serial-and-debug-

adapter:OPENSDA? code $=$ OPENSDA\&nodeId $=0152$ 109D3F1E8C1EB4, Julho 2015.

[9] Altium Limited. Engineering innovation for PCB design. http://www.altium.com/, Julho 2015.

[10] Texas Instruments Incorporated. Datasheet do CI BQ24075RGTT.

http://www.ti.com/lit/ds/symlink/bq24072.pdf , Julho 2015.

[11] Freescale Semiconductor Incorporated. Datasheet do CI MKL25Z128VLK4 http://cache.freescale.com/files/32bit/doc/ref ma nual/KL25P80M48SF0RM.pdf, Julho 2015.

[12] Nordic Semiconductor. Datasheet do módulo RF nRF24L01+ de $\quad 2,4 \quad$ GHz. www.nordicsemi.com/eng/content/download/27 26/34069/file/nRF24L01P Product Specificatio n 1 0.pdf, Julho 2015.

[13] SanDisk Corporation. Manual do micro SD card. http://media.digikey.com/pdf/Data\%20Sheets/M

z

$\underline{\text { Sys- }}$

tems $\% 20$ Inc $\% 20$ PDFs/SD $\% 20$ Card $\% 20$ Prod $\% 2$ 0Family\%20OEM\%20Manual.pdf, Julho 2015.

[14] OSRAM Opto Semiconductors. Datasheet do LED RGB. http://www.osramos.com/Graphics/XPic2/00134734 0.pdf , Julho 2015.

[15] Exar Corporation. Datasheet do CI SPX3819M5-L-3-3/TR.

http://www.exar.com/common/content/document .ashx?id=615, Julho 2015.

[16] E-radionica . Datasheet dos módulos RF de 433 $\mathrm{MHz}$. radionica.com/productdata/datasheetim120628014.pdf , Julho 2015.

[17] ON Semiconductor. Datasheet do CI NCP1400ASN50T1G. http://www.onsemi.com/pub link/Collateral/NC P1400A-D.PDF, Julho 2015.

[18] Freescale Semiconductor Incorporated. Kinetis Design Studio Integrated Development Environment

(IDE).

http://www.freescale.com/tools/embeddedsoftware-and-tools/run-time-software/kinetissoftware-and-tools/ides-for-kinetis-mcus/kinetisdesign-studio-integrated-developmentenvironment-ide:KDS IDE, Julho 2015

[19] Freescale Semiconductor Incorporated. Processor Expert Software - Integrated with CodeWarrior

Tools.

http://www.freescale.com/tools/embeddedsoftware-and-tools/software-developmenttools/processor-expert-and-embeddedcomponents/software-suites/processor-expertsoftware-integrated-with-codewarriortools:PROCESSOR-EXPERT, Julho 2015.

[20] Real Time Engineers Ltd. FreeRTOS. http://www.freertos.org/, Julho 2015.

[21] FreeRTOS real time kernel. freeRTOS, 2009.

[22] M C. Maia. CESE. http://www.michellemaia.com/cese, Julho 2015. 